\title{
Characterization of Transducers and Resonators under High Drive Levels
}

\author{
S. Sherrit, X. Bao, D.A. Sigel, M.J. Gradziel, S.A. Askins, B.P. Dolgin, and Y. Bar-Cohen \\ Jet Propulsion Laboratory, Caltech, Mail Stop 82-105, 4800 Oak Grove Dr., Pasadena, CA, 91109-8099
}

\begin{abstract}
In many applications, piezoelectric transducers are driven at $\mathrm{AC}$ voltage levels well beyond the level for which the material was nominally characterized. In this paper we describe an experimental setup that allows for the determination of the main transducer or resonator properties under large AC drive. A sinusoidal voltage from a waveform generator is amplified and applied across the transducer/resonator in series with a known high power resistor. The amplitude of applied voltage and the amplitude and the relative phase of the current through the resistor are monitored on a digital scope. The frequency of the applied signal is swept through resonance and the voltage/current signals are recorded. After corrections for the series resistance and parasitic elements the technique allows for the determination of the complex impedance spectra of the sample as a function of frequency. In addition, access to the current signal allows for the direct investigation of non-linear effects through the application of Fourier transform techniques on the current signal. Our results indicate that care is required when interpreting impedance data at high drive level due to the frequency dependence of the dissipated power. Although the transducer/resonator at a single frequency and after many cycles may reach thermal equilibrium, the spectra as a whole cannot be considered an isothermal measurement due to the temperature change with frequency. Methods to correct for this effect will be discussed. Results determined from resonators of both soft and hard PZT and a ultrasonic horn transducer are presented.
\end{abstract}

\section{INTRODUCTION}

High power density ultrasonic transducers are used in a variety of devices for drilling[1], cleaning [2], welding [3], filters 4], and novel transformers 5]. In general driving piezoelectrics at high power levels introduces nonlinear and thermal effects that need to be understood in order to accurately measure and control the transducer. In the small signal regime piezoelectrics are typically linear, isothermal, and adiabatic. When the same materials are driven at higher drive levels the thermal conditions can change considerably. In this paper we describe a test system for determining the response of the piezoelectric at and off resonance.

\section{EXPERIMENTAL}

The test system is shown in Figure 1 and allows for the simultaneous determination of the Field E, electric displacement $\mathrm{D}$, temperature $\mathrm{T}$, and the strain $\mathrm{S}$. An AC voltage is applied across the combination of the sample and an adjustable high power resistor (1-1000 $\Omega$ ). The resistor value is chosen to maximize the current signal while keeping the ratio of the voltage across the resistor to the voltage across the sample small. In low frequency measurements $(<1 \mathrm{kHz})$ a resistor $(\mathrm{R}=100-1000 \Omega)$ or a large integrating capacitor is typically used to determine the charge applied to the sample while for resonance measurements the sample impedance can be much smaller and a small high power resistor $(\mathrm{R}=1 \Omega)$ can be used.

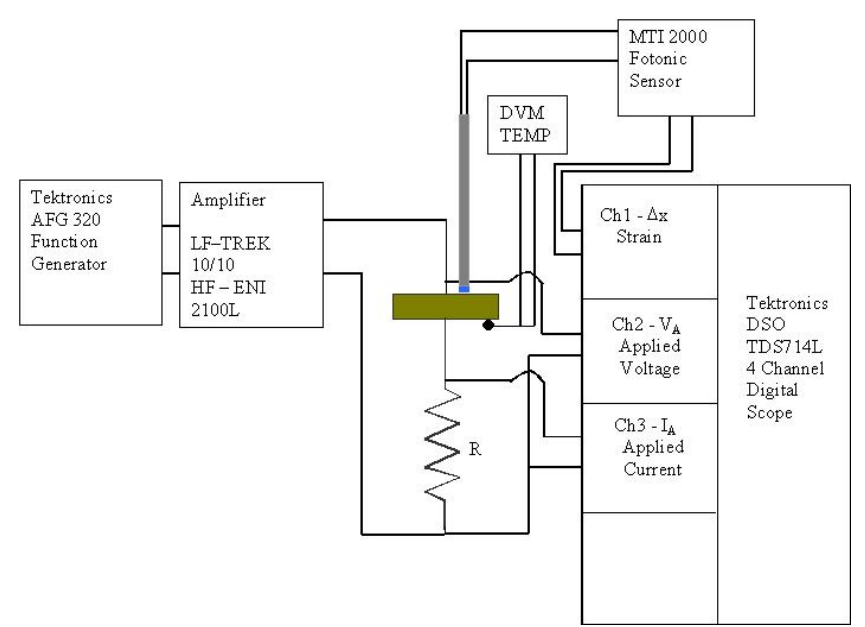

Figure 1. Experimental system to measure the electric field E, displacement $\mathrm{D}$, strain $\mathrm{S}$, temperature $\mathrm{T}$ and impedance $\mathbf{Z}$ as a function of frequency. Temperature is measured with a Chromel Alumel thermocouple.

The voltage across the resistor, displacement of the sample surface and the temperature are monitored simultaneously. The current is determined from $I=V_{R} / R$. The electric displacement is then calculated from integral of the current divided by the sample electrode area. The impedance can be determined from the ratio of the voltage and current phasors minus the current resistor R. Examples of data obtained from the system are shown in Figure 2. and 3.
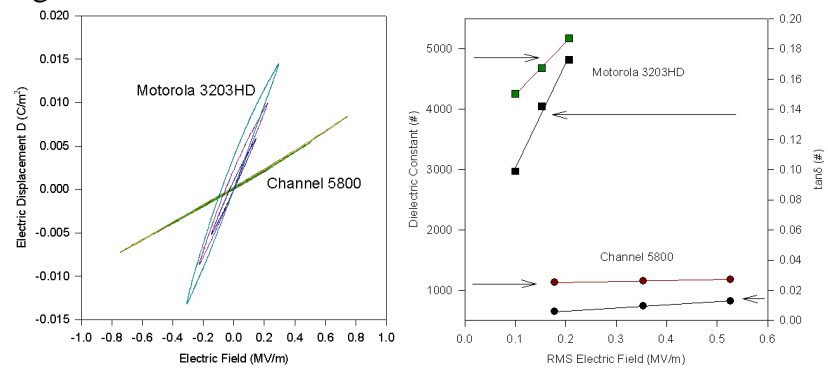

Figure 2. D-E curves and the dielectric constant and dissipation determined from the curves for a soft PZT (Motorola $3203 \mathrm{HD}$ ) and hard PZT sample (C5800). The temperature rise above ambient was less than $2{ }^{\circ} \mathrm{C}$.

A Fourier transform analysis 6 on the electric displacement data shown in Figure 2. was performed on the curves with the largest response for both the soft and hard PZT materials and the results are shown in Table 1. 

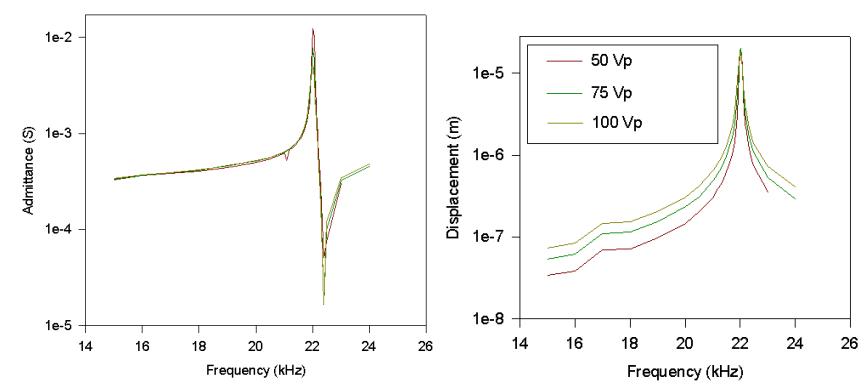

Figure 3. The admittance and tip displacement for a stepped ultrasonic horn as a function of frequency and drive level.

Table 1. Ratio of amplitudes of higher order harmonics to first harmonic for the data shown in Figure 2.

\begin{tabular}{ccc}
\hline Harmonic & Channel 5800 & Motorola 3203HD \\
\hline 1 & 100 & 100 \\
2 & 2.6 & 7.8 \\
3 & 0.59 & 3.2 \\
4 & 0.032 & 0.36 \\
\hline
\end{tabular}

The dielectric and dissipation data shown in Figure 2 is in general agreement with manufacturers specifications. The Fourier results show a larger than expected coefficient for the second harmonic, which was determined to be present, is the excitation signal. The dielectric and dissipation data in Figure 2. was calculated from the linear response. The admittance and strain of a piezoelectric horn is shown in Figure 3. for three different drive levels. The frequency was swept down in all three cases. The data shown in Figure 3 was found to agree very well with previous modeling on the horn transducer[7. The temperature measurements as a function of the drive frequency and the hysteretic behavior on sweeping up and down in frequency however was quite dramatic and unexpected as can be seen in Figure 4.

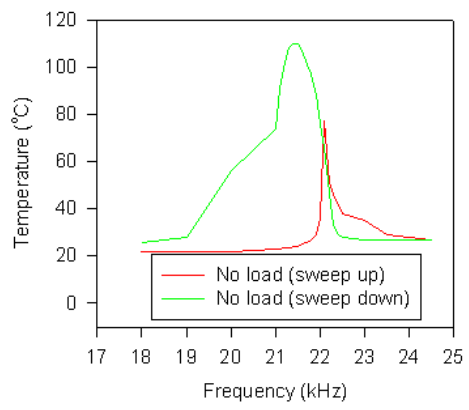

Figure 4.Thermal hysteresis of the stepped horn driven at $180 \mathrm{Vp}$.

Similar results were found for a ring resonator of Channel 5800 material as shown in Figure 5 and 6. Other authors have reported these thermal effects (hysteresis and frequency jumps[8], 9 and attribute the various effects to elastic nonlinearities. It is apparent from the data in figures 4,5 and 6 that at least part of the hysteresis and frequency jump is due to the lack of isothermal conditions during the measurement.
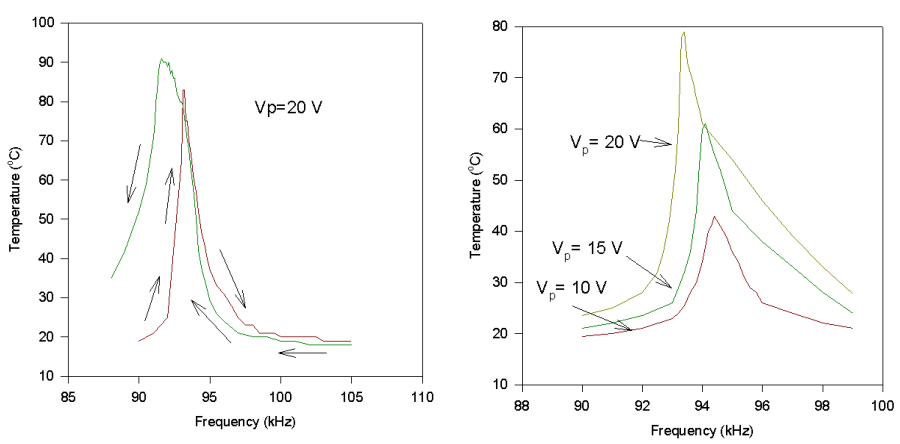

Figure 5. Temperature frequency curves and thermal hysteresis for a Channel 5800 ring sample around resonance

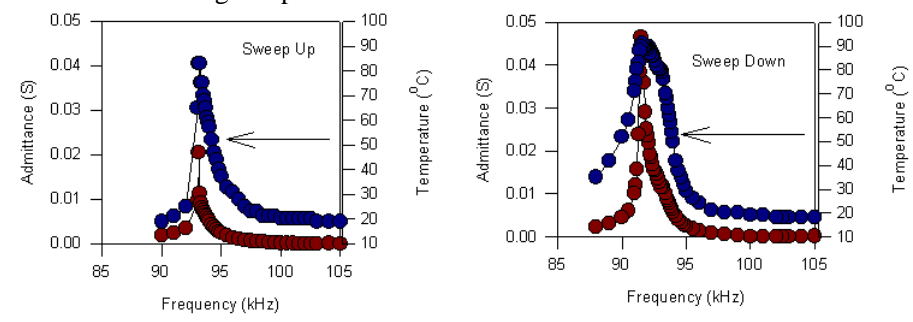

Figure 6. Thermal hysteresis and frequency jumping for a ring resonator sample of hard PZT (Channel 5800) around resonance. Frequency shift in the peak of the temperature and admittance are seen to correlate.

\section{DISCUSSION}

In order to investigate the thermal hysteresis and the frequency shifts in the admittance plots or properly evaluate the properties of the resonator one has to look at the dissipated power and the heat flows in the sample under test and the resultant temperature distribution. When driving a piezoelectric material off resonance the power dissipated in the sample is primarily due to the dielectric loss $(\tan \delta)$. The average power dissipated is

$$
P_{D}=L A \pi f E^{2} \varepsilon \tan \delta
$$

where $f$ is the frequency, $\mathrm{E}$ is the electric field amplitude and $\varepsilon$ is the real permittivity $L$ is the sample thickness and $\mathrm{A}$ is the electrode area. At frequencies below resonance the power is dissipated evenly throughout the sample. At resonance the power dissipation is primarily due to the mechanical loss and proportional to the square of the strain distribution. Since the current and voltage are in phase we can determine the average power dissipated from

$$
P_{M}=V_{R M S}^{2} Y\left(f_{S}\right)
$$

where $V_{\text {RMS }}$ is the root mean square voltage across the sample and $\mathrm{Y}\left(f_{\mathrm{S}}\right)$ is the admittance at the resonance frequency $f_{\mathrm{s}}$. Unlike the off resonance case however the power dissipation can be shown to have a spatial variation in the sample that is proportional to square of the strain distribution. For a plate sample of thickness $\mathrm{L}$ and area $\mathrm{A}$ centered at $\mathrm{x}=0$ the power distribution as a function of $\mathrm{x}$ is

$$
P(x)=2 P_{M} \cos ^{2}\left(\frac{\pi x}{L}\right)
$$


where we have normalized the power distribution between $x=-L / 2$ and $x=L / 2$ to have an average power equal to $P_{M}$ throughout the sample volume.

\section{Internal temperature of piezoelectric $\mathrm{T}_{\mathrm{i}}(\mathrm{x})$}

The internal temperature of the piezoelectric can be determined by applying the steady heat flow equation with generation.

$$
\nabla^{2} T=-\frac{q(x)}{k}
$$

where $\mathrm{q}(\mathrm{x})$ is the power dissipated per unit volume and $\mathrm{k}$ is the thermal conductivity of the material. Using equation 4 and letting $\mathrm{T}(-\mathrm{L} / 2)=\mathrm{T}(\mathrm{L} / 2)=\mathrm{T}_{1}$ and $\mathrm{q}(\mathrm{x})=\mathrm{P}(\mathrm{x})$ (equation $3)$ the internal temperature of the piezoelectric material at resonance to found to be.

$$
T(x)=\left(\frac{P_{M} L}{2 k A}\right)\left(\frac{\cos ^{2}(\pi x / L)}{\pi^{2}}-\frac{x^{2}}{L^{2}}+\frac{1}{4}\right)+T_{1}
$$

In the case where the power dissipation distribution is a constant as a function of $\mathrm{x}$ as is the case for the dielectric loss below resonance the temperature distribution can be shown to be.

$$
T(x)=\frac{P_{D}}{2 k A L}\left(\left(\frac{L}{2}\right)^{2}-x^{2}\right)+T_{1}
$$

Typical values of the thermal conductivity for PZT materials are $\mathrm{k}=1.25 \mathrm{~W} / \mathrm{m}^{\circ} \mathrm{C}$ and the heat capacity is of the order of $420 \mathrm{~J} / \mathrm{kg}^{\circ} \mathrm{C}$ [10]. It should be noted that although the surface temperature of the piezoelectric can be controlled the internal temperature rise is inherent and is required to set up the thermal gradients to conduct the dissipated power to the sample surface.

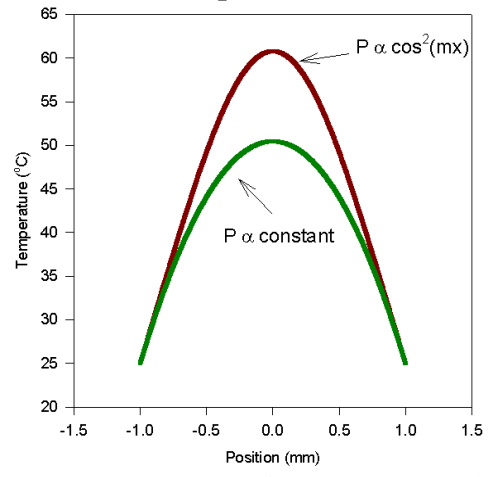

Figure 7. The internal temperature of a piezoelectric with $\mathrm{L}=0.002 \mathrm{~m}$, Dia. $=.02 \mathrm{~m}, \mathrm{k}=1.25 \mathrm{~W} / \mathrm{m}^{\circ} \mathrm{C}, \mathrm{P}=40 \mathrm{~W}$ for a constant power distribution and $\cos ^{2}(\mathrm{mx})$ distribution. Symmetric thermal boundary conditions are assumed.

\section{Surface Temperature of piezoelectric}

In order to determine the absolute temperature in the piezoelectric one must also determine the surface temperature $T_{1}$ of the sample as can be seen in equations 5 and 6. A schematic of the resonator control volume is shown in Figure 8 after thermal equilibrium has been reached. At equilibrium the flow of heat is driven by a temperature difference between the free resonator surface and the environment. The electrical power supplied by the driver is equal to the power dissipated and the power radiated

$$
\mathrm{P}_{\mathrm{E}}=\mathrm{P}_{\mathrm{D}}+\mathrm{P}_{\mathrm{R}}
$$

In a resonator operating in air the radiated power is approximately zero due to the mismatch of acoustic impedance. Under these circumstances and prior to reaching equilibrium the power dissipated can be shown 11 to be equal to

$$
P_{D}=m C_{P}(d T / d t)+d Q / d t
$$

Where $\mathrm{m}$ is the mass of the resonator, $\mathrm{C}_{\mathrm{P}}$ is the specific heat at constant pressure, $\mathrm{dT} / \mathrm{dt}$ is the rate of temperature change and $\mathrm{dQ} / \mathrm{dt}$ is the heat flow rate from the resonator to the environment through convection or conduction. At thermal equilibrium the temperature change is zero and the dissipated power is

$$
P_{D}=d Q / d t=k_{i}\left(T_{1}-T_{o}\right)
$$

equal to the heat flow. $\mathrm{T}_{1}$ and $\mathrm{T}_{\mathrm{o}}$ are the equilibrium temperature at the surface of the resonator and the ambient temperature while $\mathrm{k}_{\mathrm{i}}$ is the effective heat transfer constant and is the sum of the product of the area and the convection heat transfer coefficient and the conductive heat transfer coefficient multiplied by the conduction area divided the conduction length.

The power dissipated in the resonator can be determined from the resonator admittance and the RMS drive voltage.

$$
P_{D}(\omega)=\operatorname{Re}\left(Y(\omega,\langle T(x)\rangle) V_{R M S}^{2}(\omega)\right.
$$

where we have used $\langle\mathrm{T}(\mathrm{x})\rangle$ the average temperature in the piezoelectric in order to approximate the resonator impedance. Due to the temperature distribution in the sample the material properties are no longer homogeneous and depend on the position along the thickness direction. The actual impedance of the resonator is analogous to a material with slightly graded material properties symmetric with the centerline of the transducer. Equation 10 implies that the equilibrium temperature of the resonator surface is frequency dependent and peaks at the peak in the dissipation at $\mathrm{Y}\left(f_{\mathrm{s}}\right)$. Substituting equation 10 into 9 we find.

$$
T_{1}(\omega)=\left(\frac{\operatorname{Re}\left(Y(\omega,\langle T(x)\rangle) V_{R M S}^{2}(\omega)\right.}{k_{i}}\right)+T_{0}
$$

Under constant voltage drive and slowly increasing frequency one can determine $\mathrm{k}_{\mathrm{i}}$ the effective heat transfer constant by measuring the impedance and temperature as a function of frequency. In the case of the ring sample measured in Figure 6. it is found to be of the order of $\mathrm{k}_{\mathrm{i}} \approx 0.15 \mathrm{~W} /{ }^{\circ} \mathrm{C}$. The time constant $\tau=\rho \mathrm{L}^{2} \mathrm{C}_{\mathrm{p}} / \mathrm{k}$ for a $1 \mathrm{~mm}$ sample is of the order of 1 second. This suggests that frequency sweeps or measurement made at a single frequency should be done in fractions of seconds in order to keep the temperature constant during measurement. 


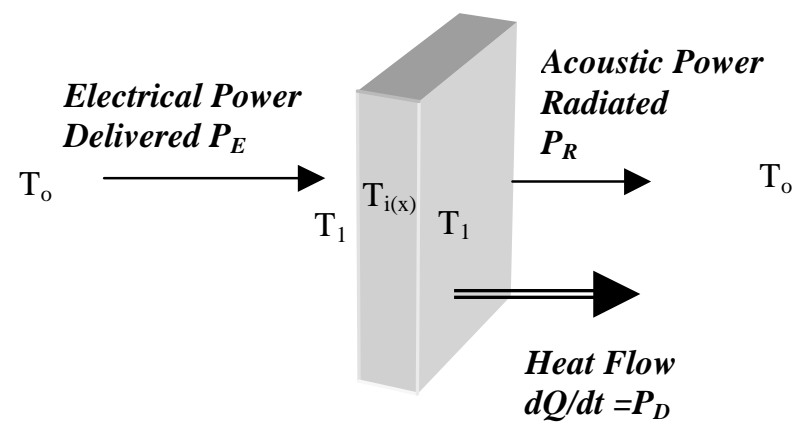

Figure 8. A schematic diagram of a transducer control volume after reaching thermal equilibrium.

Since $\langle\mathrm{T}(\mathrm{x})\rangle$ is a function of $\mathrm{T}_{1}(\omega)$ the equation shown in equation 11 is a transcendental function. Equation 11, we believe, is at least partially responsible for both the thermal hysteresis and the frequency jump in the admittance spectra. Consider approaching the resonance from below the resonance frequency $\mathrm{f}_{\mathrm{s} \text {. As the }}$ measurement frequency approaches the resonance frequency the sample begins to heat causing the resonance frequency to shift toward the measurement frequency creating an abrupt increase in the temperature, which drives the resonance frequency past the measurement frequency. Above the resonance the sample begins to cool and the decrease in admittance is more gradual. As the frequency approaches the resonance frequency from above the sample again begins to heat causing the resonance frequency to shift away from the measurement frequency creating a gradual approach to the resonance. This gives rise to a gradual increase in the temperature and admittance until it finally passes the resonance frequency and begins to cool. It is apparent that from the preceding discussion that in order to determine the material properties properly without thermal contributions the measurement must proceed very quickly or be done with single frequency steps and duty cycled to ensure minimum temperature rise in the sample

\section{CONCLUSIONS}

An experimental apparatus was presented which allows for the simultaneous measurement of the field; electric displacement, strain, impedance and temperature under high drive levels. In addition because of direct access to the current and voltage signals Fourier techniques can be used to determine nonlinear response. An alternative source of asymmetric impedance curves, thermal hysteresis and frequency jumping was identified. It is apparent from the results presented that in order to properly measure the material properties one needs to look at stepped measurements with a duty cycle to ensure isothermal conditions as was mentioned in previous work [8].

\section{ACKNOWLEDGMENTS}

The research at the Jet Propulsion Laboratory (JPL), a division of the California Institute of Technology, was carried out under a contract with the National Aeronautics Space Agency (NASA).

\section{REFERENCES}

[1]Stewart Sherrit, Yoseph Bar-Cohen, Benjamin P. Dolgin, Nathan Bridges, Xiaoqi Bao, Zensheu Chang, Albert Yen, Ronald S. Saunders, Richard A. Rainen, Stephen F. Dawson, Dharmendra Pal, Jason Kroh, Tom Peterson, "Sample Acquisition and In-Situ Analysis Using the Ultrasonic/Sonic Driller/Corer (USDC) and Robotic Platforms" Proceedings of the The $6^{\text {th }}$ International Symposium on Artificial Intelligence, Robotics and Automation in Space ( $i$-SAIRAS-01), Montreal, Canada, June 18-21, 2001

[2] J.A. Gallego-Juarez, "New Technologies in High-Power Ultrasonic Industrial Applications", Proceedings of the IEEE Ultrasonics Symposium, Cannes, France, pp. 1343-1352, 1994

[3] Jiromaru Tsujino, Tetsugi Ueoka, Tsutomu Sano, "Welding Characteristics of $27 \mathrm{kHz}$ and $40 \mathrm{kHz}$ Complex Vibration Ultrasonic Metal Welding Systems, Proceedings of the IEEE Ultrasonics Symposium, Lake Tahoe, Nevada, pp. 773-778, 1999 [4] John D. Larson, Richard C. Ruby, Paul D. Bradley, Joe Wen, Shong-Lam Kok, Allen Chien, "Power Handling and Temperature Coefficient Stucies in FBAR, Duplexers for the $1900 \mathrm{MHz}$ PCS Band, Proceedings of the IEEE Ultrasonics Symposium, San Juan Puerto Rico, pp. 869-874, 2000

[5] Torsten Bove, Wanda Wolny, Erling Ringgaard, Klaus Breboel, "New Type of Piezoelectric Transformer with Very High Power Density", Proceedings of the IEEE International Symposium on the Application of Ferroelectrics, Honolulu, Hawaii, pp.321- 324, 2000

[6] S. P. Leary, S.M. Pilgrim, "Harmonic Analysis of the Polarization Response in $\mathrm{Pb}\left(\mathrm{Mg}_{1 / 3} \mathrm{Nb}_{2 / 3}\right) \mathrm{O}_{3}$ based Ceramics- A Study in Aging" IEEE Trans. on Ultrasonics, Ferroelectrics and Frequency Control, 45, (1), pp. 163-169, 1998

[7] S. Sherrit, B.P. Dolgin, Y. Bar-Cohen, D. Pal, J. Kroh, T. Peterson "Modeling of Horns for Sonic/Ultrasonic Applications," Proceedings of the IEEE International Ultrasonics Symposium, Lake Tahoe, CA, October 1999, pp. 647-651

[8] Ralph S. Woolett, Charles L. LeBlanc, "Ferroelectric Nonlinearities in Transducer Ceramics, IEEE Trans on Sonics and Ultrasonics, SU-20, pp. 24-31, 1973

[9] Shasshank Priya, Dwight Viehland, Alfredo Vazquez Carazo, Jungho Ryu, Kenji Uchino, "High power resonant measurements of piezoelectric materials: Importance of elastic nonlinearities" Journal of Applied Physics, 90, pp. 1469-1479, 2001

[10] D. A. Berlincourt, D.R. Curran, H. Jaffe, "Chapter 3Piezoelectric and Piezomagnetic Material and their Function in Transducers, pp. 169-270, Physical Acoustics-Principles and Methods, Volume 1-Part A, ed. W.P. Mason Academic Press, New York, 1964.

[11] K. Uchino, S. Hirose, "Loss Mechanisms in Piezoelectric Ceramics: How to Measure Different Losses Separately" IEEE Trans. on Ultrasonics, Ferroelectrics and Frequency Control, 48, (1), pp. 307-321, 2001 